\title{
LAS TIC EN EL FOMENTO LECTOR DE LOS ADOLESCENTES. UN ESTUDIO DE CASO DESDE LAS CREENCIAS DOCENTES
}

\author{
Manuel Francisco Romero Oliva \\ Hugo Heredia Ponce \\ Ester Trigo Ibáñez \\ Universidad de Cádiz
}

\begin{abstract}
RESUMEN: Este trabajo, planteado como un estudio de caso desde un enfoque mixto de corte mediacional, tiene como objetivo analizar las creencias del profesorado sobre la integración de las TIC en los procesos de enseñanzaaprendizaje para el fomento de la lectura. El contexto estudiado corresponde con un centro de educación secundaria de la provincia de Cádiz. Se contó con la participación de 14 docentes $(\mathrm{N}=14)$-áreas lingüísticas $(\mathrm{n}=6)$ y no lingüísticas $(\mathrm{n}=8)$-. Para comprender la realidad analizada, fue necesaria la combinación de instrumentos cuantitativos (cuestionario de creencias y actitudes de los docentes), y cualitativos (grupos focales). Los resultados recogidos a través de una matriz DAFO, si bien reflejan una actitud proactiva del docente hacia la innovación educativa para el fomento lector del adolescente, evidencian una necesidad formativa para afrontar dicho reto desde una visión de centro educativo.
\end{abstract}

PALABRAS CLAVE: fomento lector, TIC, formación del profesorado, creencias y actitudes.

\section{THE ICT AND READING PROMOTION IN TEENAGERS. A CASE STUDY FROM THE BELIEFS OF TEACHERS}

\footnotetext{
ABSTRACT: This paper, proposed as a case study from a mixed approach of mediation, aims to analyze teachers' beliefs about the integration of ICT in teaching-learning processes for the promotion of reading. The studied context is focused in a High School center situated in Cádiz. It counted with the participation of 14 teachers $(N=14)$-linguistic areas $(n=6)$ and non-linguistic ( $\mathrm{n}=8$ )- To understand the reality analyzed, it was necessary to combine quantitative instruments (questionnaire of beliefs and attitudes of teachers), and qualitative (focus groups). The results, collected through a SWOT matrix,
} 
although they reflect a proactive attitude of the teacher towards educational innovation for the adolescent's reading promotion, show a formative need to face this challenge from a vision of an educational center.

KEYWORDS: Reading promotion, ICT, teacher's training, beliefs and attitudes.

Recibido: 14/05/2019

Aceptado: 19/07/2019

Correspondencia: Manuel Francisco Romero Oliva, Universidad de Cádiz, Facultad de Ciencias de la Educación, Campus Universitario de Puerto Real. 11510 Puerto Real, Cádiz. Email: manuelfrancisco.romero@uca.es

\section{INTRODUCCIÓN}

Actualmente existe una preocupación por la formación del profesorado y, por ello, autores, como Romero y Jiménez (2014), Bozu e Imbernón (2016), García, Rebollo y García (2016) o Trigo (2016), la resaltan: el docente precisa de una formación específica para dinamizar los procesos de formación lectora entre los adolescentes y acercarlos al disfrute desde una visión compartida y generacional. Aquí es donde aparecen las Tecnologías de la información y la comunicación -TIC, en adelanteque "pueden también ejercer efectos positivos en la sociedad en su conjunto, en áreas tales como eficiencia en la educación, entrega de servicios públicos y calidad de servicios de salud" (Katz, 2009, p. 20). De esta manera pretendemos analizar la visión -desde sus creencias y actitudes-que tiene el profesorado de un centro de la provincia de Cádiz sobre el uso de las TIC y la lectura y comprobar si la utilización de las nuevas tecnologías es idónea para conseguir atraer a nuestros nativos digitales al mundo de la lectura (Romero y Jiménez, 2014).

\subsection{Tecnologías de la información y la comunicación en la escuela}

No debemos obviar que en el mundo se están produciendo avances (Semenov, 2005) ni que nos encontramos en un continuo fluir en la sociedad de la información (Marquès, 2000a; Amar, 2008). Según la Unión General de Telecomunicaciones (2003, p. 4) es una sociedad donde "todos puedan crear, acceder, utilizar y compartir información y el conocimiento, para hacer que las personas, las comunidades y los pueblos puedan desarrollar su pleno potencial y mejorar la calidad de sus vidas de manera sostenible". Esta idea de mutabilidad y fluidez en el cambio social viene concretada por diversos autores que confeccionan una representación actual:

- la existencia de una sociedad globalizada. Los cambios que se producen actualmente no solo adquieren una dimensión en un sitio, sino que afecta a todo el mundo (Cabero, 2000), dando paso al término brecha digital (Tello, 2008; Garrido-Lora, Busquet y Munté-Ramos, 2016; Mur, 2016); 
- el uso de las TIC, definidas como "dispositivos tecnológicos (hardware y software) que permiten editar, producir, almacenar, intercambiar y transmitir datos entre diferentes sistemas de información que cuentan con protocolos comunes" (Cobo, 2009, p. 312).

Ambos aspectos confluyen en la educación pues "la introducción y el uso de las TIC en los procesos de enseñanza-aprendizaje y de gestión en la educación superior ha supuesto una transformación institucional orientada a dar respuesta a las necesidades demandadas por las sociedades del conocimiento" (Baelo y Cantón, 2009 , p. 3). Siguiendo en este paradigma emergente, la escuela precisa adaptarse a los acontecimientos y formar en el uso de estos medios desde una visión crítica y social a sus jóvenes ciudadanos para que puedan acceder y sepan utilizar estas herramientas tecnológicas (Santiago, Romero y Goenechea, 2019). De ahí surge la idea de la alfabetización digital (Marquès, 2000b; Casado, 2006; Palamidessi, 2006) que Milena, González, Torres y Araya (2017) vinculan a la capacidad de acceder a las fuentes de redes informáticas y de utilizarlas para comprender y utilizar la información.

Dicha alfabetización se hace necesaria desde la formación del docente que facilitaría la cohesión entre sus creencias y la necesidad de actualizar unas metodologías determinadas por su carácter activo y motivador desde el propio entorno del adolescente. Así, el profesorado está inmerso en un continuo dilema cuya piedra angular se asienta en la incorporación de estas herramientas en los nuevos contextos educativos. El profesorado tendrá el papel de aprendiz, pues deberá formarse en la utilización de estas herramientas:

las actitudes de los docentes hacia una metodología efectiva hacia un uso de las tecnologías, se convierten en un factor esencial para la inclusión de las TIC en los contextos educativos, pues a partir de una concepción positiva de los métodos activos y las ventajas del uso de herramientas versátiles y con beneficios pedagógicos, los docentes Ilevarán a cabo una labor de formación, dedicación de tiempo y diseño de actividades orientadas en este sentido. (Sáez, 2010, p. 264)

Además, según Palomo, Ruiz y Sánchez (2003), las TIC ofrecen posibilidades educativas, destacando el acceso inmediato a fuentes de información o la creación de recursos a través de diversas herramientas (procesadores de textos, editores de imágenes, de páginas web...). Estas vienen implementadas por la idea de Garrido-Lora, Busquet y Munté-Ramos (2016, p. 55), al incluir los aspectos relacionales de los usuarios pues los "medios de comunicación, tecnologías y redes deben considerarse no solo como tecnologías para la información y la comunicación, sino también y principalmente para la relación", con lo que las TIC integran la idea de "compartir", acotadas como Tecnologías de la Relación, la información y la Comunicación -TRIC(Gil y Marfil-Carmona, 2018).

Ante lo expuesto, nos planteamos una primera cuestión que hemos convertido en objeto de nuestro trabajo: ¿cuál es el papel que va a tener el docente ante este reto social? Las indicaciones de Barberá (2003, p. 60) sirven para centrar nuestra actuación: 
En la sociedad de la información, el profesor deja de ser considerado el único poseedor de un saber que sólo tendría que transmitir. Ahora se convierte fundamentalmente en el asociado de un saber colectivo que debe organizar y ayudar a compartir. En esta perspectiva, el proceso de enseñanza y aprendizaje se ve como un diálogo en colaboración entre elementos diversos entre los cuales destaca la voz del profesorado por su capacidad de estructurar, facilitar y guiar esta interacción.

\subsection{Lectura y mediación docente en la escuela actual}

"La lectura como base para el acceso a las diferentes competencias educativas, requiere una interrelación curricular y activación de conocimientos y experiencias personales que contribuyan a la formación integral y crítica de los escolares" (Romero y Trigo, 2015, p. 17). Tiene un carácter formativo y educativo en donde habría que rescatar el concepto de animación y fomento de la lectura pues es uno de los términos más usados en la escuela y como expresa Yubero (1996, p. 60) "es un proceso de aprendizaje intencionalmente educativo, cuyo objetivo final será la autoeducación que acercará al sujeto al tan deseado hábito lector". En consecuencia, los encargados de ello son los Ilamados mediadores (Cerrillo, Larrañaga y Yubero, 2002; Boixareu, 2014), aunque hay otros autores que lo Ilaman facilitadores (Argote y Molina 2010; Caldera, Escalante y Terán, 2010). Nosotros optamos por el término mediador, ya que entendemos que este concepto hace de puente entre dos personas unidas por un mismo objetivo: la formación de lectores y el disfrute del hecho literario en toda su dimensión. Por consiguiente, en palabras de Boixareu (2014, p. 81), "entendemos como mediaciones lectoras todas aquellas personas, grupos de personas o Instituciones que acercan, estimulan u orientan a un posible lector." Estos mediadores son claves en el proceso lector porque como expresa Sainz (2005, p. 357):

Los niños requieren un intermediario, un mediador que facilite sus primeros encuentros con los libros, que les ayude a descubrir el significado, la emoción y el gozo que encierran, que mantenga su interés en la lectura hasta que llegue a formar parte indispensable de su vida cotidiana. La afición a la lectura depende de la relación estrecha y significativa con personas que valoren y disfruten la lectura, que contagien con su entusiasmo al lector en ciernes. Sin adultos lectores en el entorno es difícil que los niños lleguen a interesarse por leer.

Nos plantemos el perfil de un mediador respecto al fomento de la lectura en las aulas y seguimos a Cerrillo, Larrañaga y Yubero (2002) en su delimitación, destacando el gozo, imaginación y creatividad, desde la propia militancia al incorporar la lectura no solo en nuestro modus docendi, sino también en nuestro modus vivendi que nos lleve al deseo por la lectura y compartirla con nuestro entorno personal y profesional; para que, como indica Martín en el periódico El País (2000):

acertemos a constárselos con convicción, transmitiéndoles ese temblor que ocultan, el sentimiento de su maravilla y de su extrañeza. Porque basta que un niño oiga hablar de un elefante verde para que al instante quiera saber más, y de ese cuento podamos pasar a otros nuevos. 
A raíz de esta cita, nos tendríamos que plantear cuáles son las funciones que deben tener estos mediadores. Cerrillo, Larrañaga y Yubero (2002, p. 230) plantean una serie de puntos que deben desempeñar, entre los cuales nosotros destacamos algunos. Por una parte, creemos que lo importante es ayudar a leer por leer, es decir, al descubrimiento de la lectura placentera, pero en primer lugar debemos trabajar la lectura intensiva para que el alumno consolide sus principios lectores y de esta forma, pueda disfrutar de ella. Además, creemos que otro de los puntos que podemos resaltar es el de coordinar la selección de lecturas por edades para facilitar la formación lectora del adolescente. $Y$, de esta forma, contribuiríamos a nuestro objetivo final como mediadores: crear y fomentar hábitos lectores estables.

Para que se consiga ese objetivo el docente debe ser la figura principal en la formación lectora, lo que algunos autores denominan animador a la lectura (Yubero, 1996). Por eso, tiene un amplio abanico de herramientas que pueden servirle para motivar y crear ese gusto lector en sus alumnos que como se ha mencionado anteriormente, en esa etapa se encuentran desmotivados. De este modo, la mediación principal está entre el adolescente y el profesorado por varios motivos: les dará oportunidades que no pueda adquirir fuera de la escuela y por lo tanto, pueda construir su conocimiento y por último, el número de horas que permanece allí: "La mediación es entendida como la interacción entre el docente y el estudiante, en esta el papel del maestro permite la circulación y construcción del conocimiento en la escuela, es desde allí como los estudiantes se vinculan con sus aprendizajes" (Milena, 2014, p. 255). Además, hay que tener en cuenta que el docente debe, primero, ayudar a aqueIlos que no saben leer y no pueden ni acercarse a la lectura y en segundo lugar, hacer que les guste leer e integren esta actividad en sus vidas (Cuevas y Marzal, 2007). En este sentido, los alumnos necesitan tener un acompañamiento y ser guiado por el docente hasta que puedan conseguir esa autonomía lectora, sustituyendo obligar e imponer por mostrar, descubrir, animar... (Zayas, 2012).

En este sentido las TIC nos ofrecen diferentes posibilidades para fomentar la lectura, como por ejemplo las Redes Sociales, los medios de comunicación que como expresan Gallar, Rodríguez, Barrios (2015, p. 161) "La instancia mediadora audiovisual ocupa en muchas ocasiones, en la vida de los niños y jóvenes, un espacio mucho más amplio y agresivo que la instancia mediadora escolar". De ahí que surja nuestra segunda pregunta de investigación: ¿pueden las TIC incorporarse en los procesos de mediación lectora en la escuela e integrarse en los modelos vivenciales de nuestros estudiantes para compartir sus preferencias lectoras? Hay estudios, como Lluch (2014), que abren una puerta a la esperanza ya que nuestros adolescentes comparten las inquietudes, impresiones o sentimientos que les producen sus libros; y estos brotes vienen refrendados por la prescripción curricular que, como indica Paredes (2005, p. 259), "muchos currículos, como el norteamericano o el español, proponen que la utilización de las TIC por los alumnos debe facilitar la búsqueda, interpretación y afianzamiento de la lectura y la escritura".

Pero, para que el docente pueda ejercer una efectiva mediación lectora utilizando las TIC como alidadas, es preciso contar con una sólida formación que le permita ir 
desarrollando su entorno personal de aprendizaje en favor de una paulatina alfabetización informacional y tecnológica que le permita acercarse al modus vivendi del adolescente. Esta razón nos hace plantearnos una tercera pregunta de investigación: ¿en qué medida influye el bagaje formativo del profesorado a la hora de plantear estrategias didácticas que ayuden a fomentar la lectura utilizando las TIC? Santos (2017) destaca la importancia de ofrecer oportunidades formativas al profesorado para que, paulatinamente, pueda ir desarrollando actuaciones encaminadas al fomento lector desde una perspectiva más actualizada. Así, como recoge Trigo (2016), en Andalucía, se ofrece al profesorado un conjunto de recursos que proporcionan seguridad para acercar la lectura a los adolescentes a través de las TIC. Entre estos recursos destacamos los siguientes:

a) Programas de innovación educativa, entre los que destacan ComunicA y el Proyecto Lingüístico de Centro (PLC). A través de estos programas se pretende que el alumnado afiance su competencia lingüística. Para ello, se prestará especial atención a la lectura desde sus enfoques intensivos, de carácter formativo, y extensivos, de carácter placentero.

b) Red Profesional de bibliotecas Escolares. Se trata de un espacio virtual a través del cual los centros escolares pueden compartir su plan de trabajo y encontrar asesoramiento por parte de personal especializado en el fomento de la lectura.

\subsection{Los adolescentes en la escuela actual}

Estos recursos, Ilamados artefactos digitales en nuestra acción educativa, (Trujillo y Conecta 13,2014, p. 28) son considerados como "un producto generado por medio de dispositivos electrónicos dentro del marco de un proyecto de aprendizaje, ya sea por los estudiantes o por el docente, como resultado de un proceso de tratamiento de la información para la construcción de conocimiento". Entonces, en la escuela hay estudiantes que saben utilizar estas herramientas porque han nacido en la era digital y otros que no. En este sentido, se produce un cambio generacional, dando lugar a las siguientes dicotomías:

Tabla 1. Diferenciación entre nativos digitales e inmigrantes digitales. Prensky (2001, citado por Cassany, 2012)

\section{NATIVOS DIGITALES}

INMIGRANTES DIGITALES

Se sienten cómodos con los documentos hi- Menos facilidad para manipular imágenes y pertextuales y multimodales vídeos

Practican la multitarea Practican la monotarea

Se conectan a la red siempre que pueden y Nos incomoda el hipertexto son cooperativos

Están acostumbrados al intercambio rápido, a los textos brees y a las respuestas inmediatas

Aprenden de manera informal o incluso ju- Familiarizados con los escritos largos y con gando las respuestas dilatadas 
Frente a esta clasificación, Kruse (2010 citado por Cassany, 2012) realiza otra: residentes y visitantes digitales basada más en las motivaciones que tienen los miembros de la red para acceder a ella, es decir, por la permanencia que tienen en internet. Por lo tanto, no depende de sus edades como las anteriores.

Tabla 2. Diferenciación entre visitantes y residentes. Kruse (2010, citado por Cassany, 2012)

\begin{tabular}{ll}
\hline \multicolumn{1}{c}{ VISITANTES } & \multicolumn{1}{c}{ RESIDENTES } \\
\hline Se conecta de vez en cuando a la red & Viven en la red \\
\hline $\begin{array}{l}\text { Se conecta para revisar el correo o buscar al- } \\
\text { gún dato po comunican con sus amigos por Twitter y }\end{array}$ & WhatsApp \\
\hline Cree que la red es algo añadido, virtual o di- & Cuelgan las fotos en Picasa y los videos en \\
ferente de la realidad & YouTube \\
\hline & Actualizan sus perfiles en Linkedln y Facebook \\
\hline
\end{tabular}

Hay otra clasificación anónima (citada por Cassany,2012) que diferencia entre consumidores y productores, basada más en que los usuarios modifiquen en línea sus perfiles y por lo tanto, sean "los creadores" o no, que es lo que la diferencia de la anterior clasificación.

Tabla 3. Diferenciación entre consumidores y productores. Anónima (citada por Cassany, 2012)

\begin{tabular}{ll}
\hline \multicolumn{1}{c}{ CONSUMIDORES } & \multicolumn{1}{c}{ PRODUCTORES } \\
\hline $\begin{array}{l}\text { Les interesa las redes sin hacer aportaciones } \\
\text { personales }\end{array}$ & $\begin{array}{l}\text { Tienen perfiles sociales cargados y actualiza- } \\
\text { dos }\end{array}$ \\
\hline $\begin{array}{l}\text { Entran en chat y husmean las conversaciones } \\
\text { y los perfiles, pero no escriben }\end{array}$ & $\begin{array}{l}\text { Comparten música preferida en Spotify. Ade- } \\
\text { más, suben los PowerPoint a Slideshare o los } \\
\text { textos a Scribd }\end{array}$ \\
\hline $\begin{array}{l}\text { Aprovechan todo de El Rincón del Vago, pero } \\
\text { sin aportar. Al igual que miran recomendacio- }\end{array}$ & $\begin{array}{l}\text { Tuitean sus impresiones sobre una conferen- } \\
\text { nes y postean periódicamente en el blog de } \\
\text { nestaurantes y hoteles }\end{array}$ \\
\hline
\end{tabular}

En este sentido, vemos necesario comentar las diferentes estrategias o líneas de actuación para la formación del profesorado de hoy en día referente a la lectura y las TIC.

\section{Metodología}

Esta investigación tiene como objeto de estudio un centro educativo (Stake, 1995). Se utiliza una metodología mixta de corte mediacional, siendo el centro de interés la figura del docente desde su perspectiva cognitiva -pensamiento y creencias docentes- y técnica -aspectos de planificación y desarrollo de su acción educativa-, y en la que el investigador se convierte en el principal activo en la recogida de datos (Colás, 1998), mediante diversas técnicas e instrumentos vincula- 
dos a unos objetivos específicos que contribuirán a la comprensión de la situación (Sadín, 2003):

- Objetivo 1: analizar las creencias del profesorado sobre la integración de las TIC en los procesos de enseñanza-aprendizaje para el fomento de la lectura. Este objetivo se convierte en el eje central de la investigación pues, como expresan Cassany y Llach (2017, p. 96), "la actuación del profesorado es consecuencia de sus creencias, en el sentido de que la predicen y determinan", es decir, cómo son sus prácticas en cuanto a la lectura y la integración de las TIC en sus clases.

A partir de este objetivo, se desglosan otros tres objetivos:

- Objetivo 2: analizar las aportaciones de la TIC en las dinámicas lectoras desde la concreción de sus propias actitudes. El interés de este objetivo se corresponde con las palabras de Bautista y Méndez (2015, p. 98): "la introducción de las TIC en los ambientes educativos transforma las formas de leer y escribir, los ambientes de aprendizaje y los tipos de textos que se leen, al tiempo que se producen $-y$, sobre todo, se complejizan y diversifican- nuevos modos de leer".

- Objetivo 3: estudiar la adaptación del profesorado a la nueva cultura digital al ser "imprescindible asociar la cultura digital con la formación del profesor, puesto que se considera que el profesional de la educación está anclado a su proceso formativo, en el que se construye la intencionalidad de la profesión" (Duso y Cerutti, 2017, p. 208).

- Objetivo 4: elaborar una matriz DAFO en la que se sinteticen los aspectos contextuales de la investigación, desde los aspectos que contribuyen o ejercen dificultades para la integración de las TIC en las planificaciones docentes para la formación de lectores adolescentes.

Los objetivos de la investigación se concretaron en el diseño de una metodología mixta, que combinó instrumentos cuantitativos (cuestionario de creencias y actitudes de los docentes, objetivos 1 y 2), y cualitativos (grupos focales y matriz DAFO, objetivos 3 y 4) con la intención de comprender la realidad analizada.

\section{Contexto y participantes}

Este estudio se ha realizado con el profesorado de un centro educativo de educación secundaria obligatoria, situado en la localidad de Rota (Cádiz), y se integra en una investigación superior como fue una tesis doctoral. Los sujetos de la investigación se corresponden con 14 docentes tanto de las áreas lingüísticas (AL: 6 docentes) como no lingüísticas (ANL: 8 docentes).

\section{Técnicas e instrumentos}

Para llevar a cabo esta investigación se han utilizado diversos instrumentos que nos ayudarán a conseguir nuestros objetivos: 
a. Cuestionario: Este instrumento de análisis, Creencias del profesorado acerca de las TIC y la lectura en el aula, (Heredia y Romero, 2019, pp. 61-63), se compone de 18 preguntas o ítems con formatos diversos (cerrado, abierto y respuesta múltiple) que se distribuyeron en cinco dimensiones:

- Dimensión 1 (ítems 1, 2, 3), en el que se detecta el perfil docente desde su experiencia y área de impartición docente.

- Dimensión 2 (ítems 4 y 5), donde se busca conocer la concepción y creencias de estos docentes respecto a las TIC.

- Dimensión 3 (ítems 6, 7, 8, 9), centrada en detectar la presencia de las TIC en el aula y la finalidad educativa.

- Dimensión 4 (ítems 10,11, 12, 13), repercusiones metodológicas de la incorporación de las TIC así como las necesidades formativas requeridas para ello.

- Dimensión 5 (ítems 14, 15, 16, 17), implicación del docente en el fomento de la lectura y su conexión con las TIC.

b. Grupos focales. Como expresan Groos y Stiller $(2015$, p. 6) nos sirven para "para obtener información de aspectos relacionados con temas de investigación, al tomar en cuenta la experiencia directa de los involucrados". Los testimonios aparecen codificados con el número de informante entre paréntesis (INF_01... INF_14).

c. Matriz DAFO. Tras el análisis y categorización de los resultados obtenidos en esta investigación, se procedió a diseñar esta matriz -figura 1- con la intención de analizar las debilidades, amenazas, fortalezas y oportunidades que nos pueden ofrecer las TIC y la lectura en cuanto a la formación del profesorado de hoy en día.

\begin{tabular}{c|c}
\hline Debilidades & Amenazas \\
\hline & \\
\hline Fortalezas & Oportunidades \\
\hline & \\
\hline
\end{tabular}

Figura 1. Estructura de la matriz DAFO

El tratamiento de los datos se realizó mediante dos programas informáticos: Statistical Package for the Social Sciences (SPSS v.20), para los datos cuantitativos; y Nvivo11, para el análisis cualitativo. 


\section{Resultados}

El análisis de las dimensiones de estudio del cuestionario del profesorado ofreció una primera imagen sobre el concepto que les sugieren las TIC -Dimensión 1-. La ingeniería de datos mediante conglomerados con el software NVivo 11 facilitó el estudio de las respuestas a través de nubes de palabras -figura $2-$.

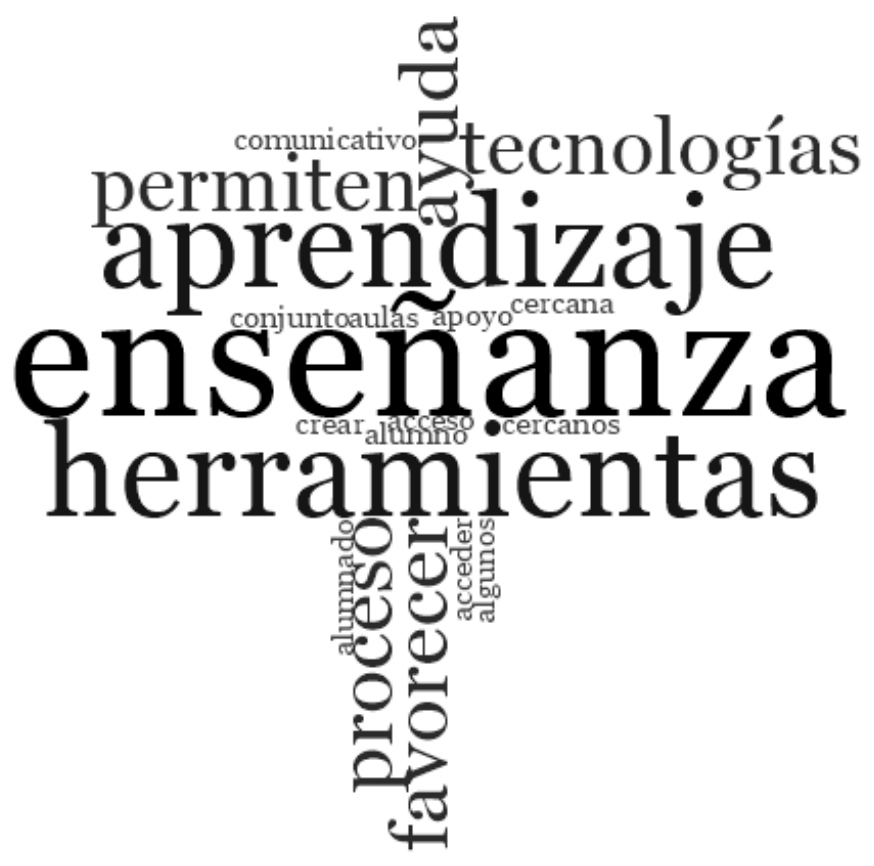

Figura 2. Palabras que aparecen en las definiciones

Como se ha podido observar en las definiciones elaboradas por los profesores en el ítem 2, destacan los términos enseñanza, herramientas y aprendizaje. De esta forma, la tendencia se consolida en dos líneas bien delimitadas: para unos, representan "un conjunto de herramientas que facilitan el acceso a la información y que nos permiten crear un entorno comunicativo. En la docencia, están al servicio del profesorado para favorecer métodos de enseñanza/ aprendizaje más cercanos al alumnado" (INF_3); o, bien, se convierten en "herramientas o recursos tecnológicos para favorecer el proceso de enseñanzaaprendizaje". (INF_10).

Estas concepciones nos pueden servir para conectar con los resultados del ítem 4 del cuestionario y determinar qué palabras les sugerían las TIC desde la confección de dos campos gnoseológicos -figura 3- referidos a sus propias concepciones docentes, tanto positivas como negativas: 
Palabras positivas

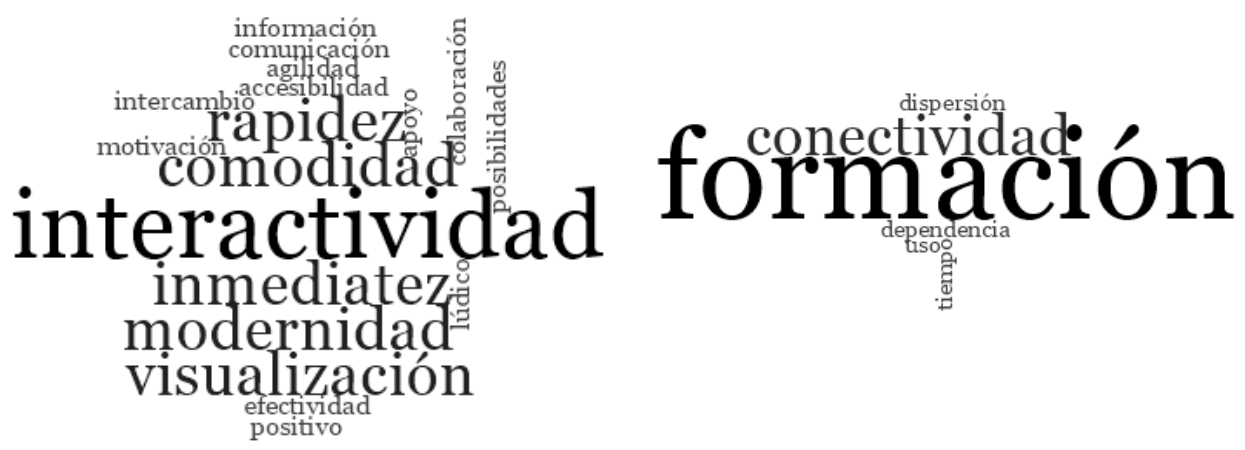

Figura 3. Palabras positivas y negativas sobre las TIC

Se puede apreciar que las TIC, como recurso positivo, fueron caracterizadas por su interactividad, inmediatez o comodidad a la hora de acceder a cualquier recurso en el aula, aspectos todos que se vinculan a la motivación y recursividad educativa; sin embargo, frente a estos rasgos, los docentes también destacaron un problema: su formación, que se convirtió en una necesidad a la hora de plantearse su uso. Este aspecto se unió a la idoneidad de los medios y su funcionamiento desde los problemas de conectividad que presentaba el centro.

La repercusión de la formación en la incorporación de las TIC se concretó con el análisis de las respuestas ofrecidas por los ítems 12 y 13 -Dimensión 4- donde se les preguntaba sobre las necesidades formativas. Ante esta situación, el $100 \%$ del profesorado expuso la necesidad de una formación para la utilización de estas en el aula y, de esta forma, estar al día para preparar las clases. Podemos observar -tabla 4- que el $85.7 \%$ de los profesores encuestados del centro habían realizado formación en TIC.

Tabla 4. Formación del profesorado sobre las TIC en el aula

\begin{tabular}{|l|c|c|c|c|}
\hline \multicolumn{1}{|c|}{ ÍTEMS } & Frecuencia & Porcentaje & $\begin{array}{c}\text { Porcentaje } \\
\text { válido }\end{array}$ & $\begin{array}{c}\text { Porcentaje } \\
\text { acumulado }\end{array}$ \\
\hline No & 2 & $14.3 \%$ & $14.3 \%$ & $14.3 \%$ \\
\hline Sí & 12 & $85.7 \%$ & $85.7 \%$ & $100 \%$ \\
\hline Total & 14 & $100 \%$ & $100 \%$ & \\
\hline
\end{tabular}

Dicha formación fue muy diversa entre los que la desarrollaron. Esta oscilaba entre la autoformación y la formación entre compañeros, más propia de la autogestión del propio centro educativo; y la formación externa con predominio de la asesoría del centro de profesorado de referencia (CEP) y los cursos on line. De esta forma, 
siendo conscientes de dicha necesidad formativa, desde la dimensión 2 resultaba necesario concretar el uso de las TIC en sus actuaciones docentes -ítem 6-. El índice de frecuencia -tabla 5- mostró una tendencia 57.1\% al empleo semanal de las TIC en aulas, siendo un porcentaje muy bajo el que solo las utilizaba con una frecuencia mensual y, por tanto, les otorgaba un carácter residual en su planificación docente.

Tabla 5. Frecuencia de las TIC en el aula

\begin{tabular}{|l|c|c|c|c|}
\hline \multicolumn{1}{|c|}{ ÍTEMS } & Frecuencia & Porcentaje & $\begin{array}{c}\text { Porcentaje } \\
\text { válido }\end{array}$ & $\begin{array}{c}\text { Porcentaje } \\
\text { acumulado }\end{array}$ \\
\hline Una vez al mes & 2 & $14.3 \%$ & $14.3 \%$ & $14.3 \%$ \\
\hline $\begin{array}{l}\text { Una vez a la } \\
\text { semana }\end{array}$ & 8 & $57.1 \%$ & $57.1 \%$ & $71.4 \%$ \\
\hline $\begin{array}{l}\text { En todas las } \\
\text { sesiones }\end{array}$ & 4 & $28.6 \%$ & $28.6 \%$ & $100 \%$ \\
\hline Total & 14 & $100 \%$ & $100 \%$ & - \\
\hline
\end{tabular}

La finalidad de la incorporación de las TIC -ítem 8- evidenció en el porcentaje de casos de la tabla 6, que estos docentes las utilizaban para preparar las clases $(85.7 \%)$ desde la idea de innovación educativa $(71.4 \%)$ y el fomento de la motivación de los estudiantes (85.7\%). Sin embargo, un aspecto, como interactuar con el mundo de los adolescentes, quedó relegado al último lugar en las preferencias educativas $(17.1 \%)$.

Tabla 6. Utilidad de las TIC

\begin{tabular}{|l|c|c|c|}
\hline \multicolumn{1}{|c|}{ ÍTEMS } & Frecuencia & Porcentaje & Porcentaje de casos \\
\hline Preparar las clases & 12 & $29.3 \%$ & $85.7 \%$ \\
\hline $\begin{array}{l}\text { Fomentar la participación en el } \\
\text { alumnado }\end{array}$ & 12 & $29.3 \%$ & $85.7 \%$ \\
\hline $\begin{array}{l}\text { Cambiar la metodología e } \\
\text { innovar }\end{array}$ & 10 & $24.4 \%$ & $71.4 \%$ \\
\hline $\begin{array}{l}\text { Acercarme al mundo de los } \\
\text { alumnos }\end{array}$ & 7 & $17.1 \%$ & $50.0 \%$ \\
\hline
\end{tabular}

De ahí se desprende que el profesorado indicara que el cambio de metodología con la incorporación de las TIC repercutía enormemente en el alumnado, ya que de esta forma pueden entender mejor los contenidos de las diferentes materias: "Hace más dinámicas las clases. El alumnado se siente más protagonista y es más participativo" (INF_10); "Organizo las clases con PowerPoint e intento fomentar la participación" (INF_14). Para tal fin, en el ítem 9 se ofrecían una serie de herramientas para analizar los recursos que empleaban -tabla 6-. Los recursos TIC que más utilizaban -tabla 7- correspondían a Google (29.8\%), el blog educativo (14.9\%) y Youtube o programas como PowerPoint (12.8\% respectivamente). Todos ellos, con un marcado carácter intuitivo y de gran difusión entre los usuarios. 
Tabla 7. Recursos TIC utilizados por el docente

\begin{tabular}{|l|c|c|c|}
\hline \multicolumn{1}{|c|}{ ÍTEMS } & Frecuencia & Porcentaje & Porcentaje de casos \\
\hline Google & 14 & $29.8 \%$ & $100 \%$ \\
\hline Blog & 7 & $14.9 \%$ & $50 \%$ \\
\hline Youtube & 6 & $12.8 \%$ & $42.9 \%$ \\
\hline PowerPoint & 6 & $12.8 \%$ & $42.9 \%$ \\
\hline Wiki & 5 & $10.6 \%$ & $35.7 \%$ \\
\hline Redes Sociales & 3 & $6.4 \%$ & $21.4 \%$ \\
\hline Plataforma educativa & 3 & $6.4 \%$ & $21.4 \%$ \\
\hline Actividades interactivas & 1 & $2.1 \%$ & $7.1 \%$ \\
\hline Simuladores & 1 & $2.1 \%$ & $7.1 \%$ \\
\hline Compiladores & 1 & $2.1 \%$ & $7.1 \%$ \\
\hline
\end{tabular}

Según la distribución de respuestas ofrecidas -tabla 7-, el porcentaje de casos indica que la utilización de herramientas como Google fue utilizada por todos los docentes (100\%), para buscar información sobre algunos temas. Como segundo recurso se posiciona el blog (50\%), con la idea de llevar un diario de clase con las actividades de todo tipo según las materias y anticipar lo que se va a impartir en la clase. Los recursos relacionados con la búsqueda de información suponen un 36.2 \% del porcentaje de frecuencia y se concentran en tres herramientas. En ellos, el porcentaje de casos es similar pues oscila en un número de 6 profesores que lo utilizaban: YouTube, para buscar algún contenido de la asignatura o visualizar fragmentos de series o películas; el programa PowerPoint, para hacer más dinámicas las clases; y las Wiki, como plataforma para compartir información académica. Son prácticas informacionales que cada vez se van haciendo más comunes en su planificación y uso para aspectos académicos. Destaca el bajo índice de uso de las redes sociales $(6.4 \%$ y de la plataforma educativa (6.4\%) como recursos para compartir aspectos académicos.

Así, ante la alta repercusión en los procesos de enseñanza/aprendizaje -dimensión $3-$, se les preguntó en qué aspectos de la metodología ha repercutido el uso de las TIC ítem 10- e indicaron que en el alumnado: "Más atención por parte del alumnado, ya que llega a más alumnos que hacen que entiendan mejor los contenidos de la materia. Hacer una metodología afectuosa y atractiva" (INF_11); "En la motivación del alumnado y en la agilidad" (INF_04); "Inmediatez a la hora de buscar algo que surge en clase en relación al tema que estudiamos. Se capta mejor la atención del alumnado" (INF_13).

Además, cuando se les propuso que indicaran qué aspectos del proceso de enseñanzaaprendizaje habían cambiado con el uso de las TIC -ítem 10-, sus respuestas estuvieron centradas en el alumnado y la motivación para el aprendizaje: "Hace más dinámicas las clases. El alumnado se siente más protagonista y es más participativo" (INF_02); "He logrado motivación del alumnado que no le gusta o le resulta difícil la memorización y razonamiento" (INF_12): "Mejorar las competencias educativas como el aprender a aprender, la competencia digital" (INF_13). Por ello, resultó de gran interés el análisis cualitativo de 
las concepciones docentes respecto a las TIC mediante un estudio de conglomerado por similitud de términos. Tras haber etiquetado sus respuestas con los tres elementos que más identificaban a las TIC en los procesos educativos -facilitador, interacción y motivacióncomo agentes favorecedores del proceso de enseñanza-aprendizaje -figura 4-:

\section{Nodos conglomerados por similitud de codificación}

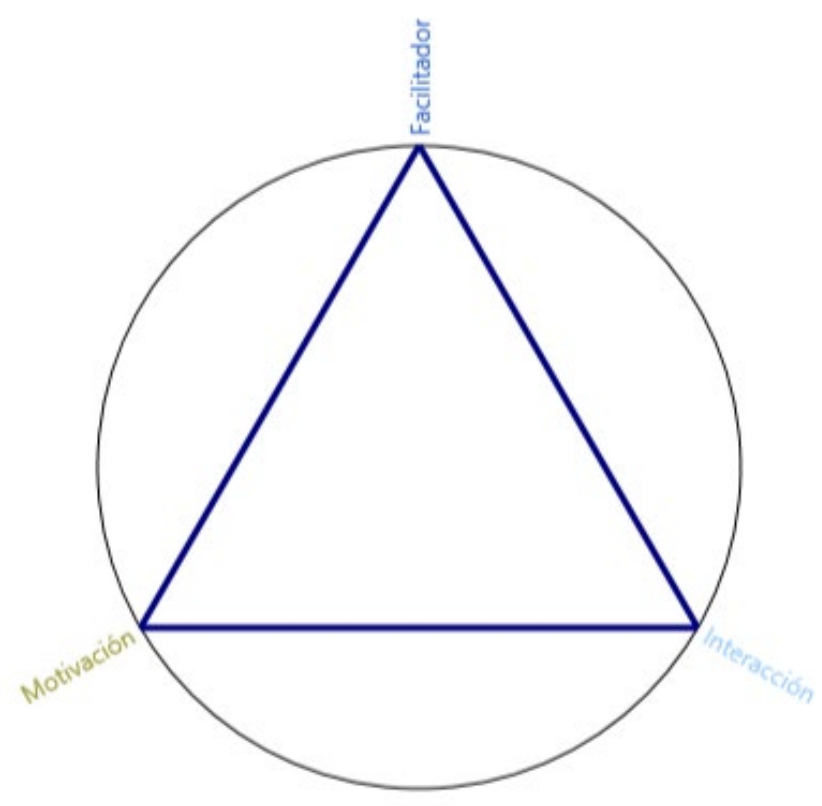

Figura 4. Conglomerado por similitud de codificación

Esta distribución se interrelaciona mediante dos nodos que tienen como referencia el coeficiente de Jaccard -tabla 8-:

Tabla 8. Análisis coeficiente de Jaccard

\begin{tabular}{|l|c|c|}
\hline \multicolumn{1}{|c|}{ Nodo A } & Nodo B & Coeficiente de Jaccard \\
\hline Nodos $\backslash \backslash$ Interacción & Nodos $\backslash \backslash$ Facilitador & 1 \\
\hline Nodos $\backslash \backslash$ Motivación & Nodos $\backslash \backslash$ Facilitador & 1 \\
\hline Nodos $\backslash \backslash$ Motivación & Nodos $\backslash \backslash$ Interacción & 1 \\
\hline
\end{tabular}

Se puede observar que la serie de estos nodos de correlación por conglomerados -tabla 9- presenta un índice con valor máximo en el coeficiente de Jaccard, distri- 
buyendo la similitud entre dos nodos o conjunto de elementos (entre 0 , como valor mínimo, y 1, como máxima similitud). De esta forma, se verifica cómo la motivación, la interacción y el factor facilitador están intrínsecamente ligados en la concepción docente de las TIC y su idoneidad para los procesos de enseñanza-aprendizaje.

Una vez analizadas las actitudes y creencias de los docentes sobre las TIC en general, procedía ahora analizar su contribución educativa al fomento de la lectura en particular -dimensión 5-. La primera delimitación fue conocer el grado de implicación en torno al desarrollo de prácticas lectoras -ítem 14-. La respuesta fue clara: 12 docentes $(85.7 \%$ ) respondieron afirmativamente; mientras que 2 (14.3\%), lo hicieron negativamente ya que pertenecían a áreas no lingüísticas (ANL). Estos fueron considerados valores perdidos en el resto de respuestas.

Tras ver que tenían planificada la lectura entre sus actuaciones docentes, se les preguntó cuánto tiempo y a qué tipo de lectura se referían -ítem 15-. Un 71.4 \% le dedica una vez a la semana, lo que viene a coincidir con la prescripción curricular que establece la necesidad de incluir un tiempo mínimo al desarrollo de la lectura en todas las asignaturas. Frente a este grupo mayoritario, un $14.3 \%$ solo la planifica en momentos puntuales -tabla 9-, Ilamando la atención que ningún docente concibiera dedicar en todas las sesiones de sus clases un tiempo a la lectura.

Tabla 9. Tiempo de dedicación a la lectura

\begin{tabular}{|c|c|c|c|c|}
\hline ÍTEMS & $\begin{array}{c}\text { Frecuencia } \\
\text { AL ANL }\end{array}$ & Porcentaje & $\begin{array}{c}\text { Porcentaje } \\
\text { válido }\end{array}$ & $\begin{array}{l}\text { Porcentaje } \\
\text { acumulado }\end{array}$ \\
\hline En todas las sesiones & 0 & $0 \%$ & $0 \%$ & $0 \%$ \\
\hline En momentos puntuales & 0 & $14.3 \%$ & $16.7 \%$ & $16.7 \%$ \\
\hline Una vez a la semana & 6 & $71.4 \%$ & $83.3 \%$ & $100 \%$ \\
\hline Total & 12 & $85.7 \%$ & $100 \%$ & \\
\hline Perdidos & 2 & $14.3 \%$ & & \\
\hline Total & 14 & $100 \%$ & & \\
\hline
\end{tabular}

Respecto a la distribución de la frecuencia entre las AL y las ANL -tabla 9-, se observa que todos los docentes de $\mathrm{AL}$ al menos dedican una sesión semanal a la lectura mientras que solo el 50 \% de las ANL también lo hace, quedando de manera residual en dos profesores que la planifican en momentos puntuales y que se unen a los otros dos que no la incluían.

Avanzando en la delimitación de la lectura -ítem 16-, las tendencias no fueron uniformes -tabla 10-; sin embargo, sí existe un predominio de la lectura desde un enfoque académico (50 \%) frente a los que prefieren dedicar su tiempo al disfrute de la lectura (14.3\%). Se observa que ningún profesor de ANL fomenta la lectura placentera y esta queda adscrita a los de AL que, además, contribuyen a todas las modalidades. En su conceptualización se tiende al desarrollo de la competencia lectora frente a la competencia literaria y desarrollo del pensamiento crítico de los estudiantes. 
Tabla 10. Tipo de lectura que trabaja el profesorado

\begin{tabular}{|c|c|c|c|c|}
\hline ÍTEMS & $\begin{array}{l}\text { Frecuencia } \\
\text { AL ANL }\end{array}$ & Porcentaje & $\begin{array}{c}\text { Porcentaje } \\
\text { válido }\end{array}$ & $\begin{array}{l}\text { Porcentaje } \\
\text { acumulado }\end{array}$ \\
\hline Lectura placentera & 20 & $14.3 \%$ & $16.7 \%$ & $16.7 \%$ \\
\hline Lectura académica & 1 & $50 \%$ & $58.3 \%$ & $75 \%$ \\
\hline Ambas & 0 & $21.4 \%$ & $25 \%$ & $100 \%$ \\
\hline Total & 12 & $85.7 \%$ & $100 \%$ & \\
\hline Perdidos & 2 & $14.3 \%$ & & \\
\hline Total & 14 & $100 \%$ & & \\
\hline
\end{tabular}

Finalmente, quedaba conocer las aportaciones de las TIC al desarrollo de la lectura -ítem 17-. En este sentido, la distribución de las respuestas se realizó de manera equitativa -tabla 11-: el 50 \% se mostró a favor o en contra respecto al uso de las TIC para trabajar la lectura. Sin embargo, en la distribución de resultados podemos observar cómo son las AL las que presentan mayor frecuencia de respuestas afirmativas (5) frente a las ANL (1). Esta tendencia se invierte en las respuestas negativas en donde las AL solo tienen una respuesta, quedando el grueso de la frecuencia en las ANL (5).

Tabla 11. Utilización de las TIC para trabajar la lectura

\begin{tabular}{|c|c|c|c|c|}
\hline ÍTEMS & $\begin{array}{c}\text { Frecuencia } \\
\text { AL ANL }\end{array}$ & Porcentaje & $\begin{array}{c}\begin{array}{c}\text { Porcentaje } \\
\text { válido }\end{array} \\
\end{array}$ & $\begin{array}{l}\text { Porcentaje } \\
\text { acumulado }\end{array}$ \\
\hline No & 15 & $42.9 \%$ & $50 \%$ & $50 \%$ \\
\hline Sí & 5 & $42.9 \%$ & $50 \%$ & $100 \%$ \\
\hline Total & 12 & $85.7 \%$ & $100 \%$ & \\
\hline Perdidos & 2 & $14.3 \%$ & & \\
\hline Total & 14 & $100 \%$ & & \\
\hline
\end{tabular}

El análisis de las dimensiones de estudio son el punto de arranque para las futuras discusiones. Desde esta perspectiva, planteamos una referencia de Cabero (2005) donde se recoge el verdadero problema de la integración de las TIC en el aula, en general, y que puede hacerse extensible a la lectura, en particular:

Es importante tener presente que los problemas hoy para su incorporación no son tecnológicos, ya contamos con unas tecnologías sostenibles y con estándares aceptados, que nos permiten realizar diferentes tipos de cosas, y con unos parámetros de calidad y fiabilidad notablemente aceptables. Los problemas posiblemente vengan en saber qué hacer, cómo hacerlo, para quién y por qué hacerlo. (p. 7)

\section{Discusiones}

En este trabajo hemos realizado un estudio sobre las creencias y actitudes que los docentes de un centro educativo plantean respecto al uso de las TIC en el fomento de la 
lectura. Abrimos un foro de discusión con una matriz DAFO, elaborada a partir de los resultados obtenidos, que nos permitirá determinar, desde una perspectiva interna, las debilidades y fortalezas; y, desde una perspectiva externa, las amenazas y oportunidades sobre la implementación de las TIC como estrategia educativa en la formación de lectores adolescentes. Siendo conscientes de que el profesorado ha indicado que la incorporación de estas herramientas está vinculada a la idea de innovación para afrontar los nuevos retos de la escuela ante la sociedad, precisamos revisar los resultados respecto a las dimensiones de estudio que se han Ilevado a cabo. Por tanto, a partir de aquí planteamos una matriz DAFO -figura 5- sobre la lectura y las TIC desde la visión del profesorado:

\begin{tabular}{|c|c|}
\hline Debilidades & Amenazas \\
\hline $\begin{array}{l}\text { - La presencia de las TIC está fuertemente } \\
\text { relacionada con prácticas letradas basadas } \\
\text { en el acceso a la información de los con- } \\
\text { tenidos de asignaturas (dimensión 3). Hay } \\
\text { poca significatividad de estrategias basadas } \\
\text { en compartir desde las Redes Sociales, lo } \\
\text { cual facilitaría su incorporación a su mo- } \\
\text { dus vivendi. } \\
\text { - Existe una tendencia de vincular la lectura } \\
\text { con aspectos académicos en ANL, lo cual } \\
\text { relega la formación de lectores placenteros } \\
\text { a las AL (dimensión 5). } \\
\text { - La formación docente no se contempla } \\
\text { desde una visión de centro educativo sino } \\
\text { como actuaciones individuales (dimen- } \\
\text { sión 4). } \\
\text { - Los problemas técnicos y la escasez de re- } \\
\text { cursos tecnológicos en las aulas dificultan } \\
\text { la incorporación de las TIC en los procesos } \\
\text { de enseñanza (dimensiones } 2 \text { y 3). }\end{array}$ & $\begin{array}{l}\text { - Concepción apriorística de que lectura y } \\
\text { nuevas tecnologías son contrarias e incom- } \\
\text { patibles, lo que supone una estigmatiza- } \\
\text { ción en aspectos de planificación docente } \\
\text { (dimensión 2). } \\
\text {-La cultura de aprendizaje no facilita una } \\
\text { actitud hacia una visión transdisciplinar de } \\
\text { la competencia en comunicación lingüísti- } \\
\text { ca desde un enfoque transversal, otorgando } \\
\text { aspectos del disfrute lector a las AL y que- } \\
\text { dando el carácter instrumental de la lengua } \\
\text { para acceder a la información y transmitirla } \\
\text {-enfoque academicista- (dimensión 1). } \\
\text {-Los libros de texto no se presentan como } \\
\text { una base de referencia para afrontar la edu- } \\
\text { cación literaria y una formación lectora } \\
\text { desde planteamientos innovadores en los } \\
\text { que las TIC formen parte de un replantea- } \\
\text { miento didáctico (dimensión 1). }\end{array}$ \\
\hline $\begin{array}{c}\text { Fortalezas } \\
\end{array}$ & Oportunidades \\
\hline $\begin{array}{l}\text { - Conciencia de los docentes respecto a la im- } \\
\text { portancia de las TIC en los procesos de moti- } \\
\text { vación y facilitador de cara a los adolescentes } \\
\text { (dimensión 2). } \\
\text { - Actitud proactiva de los docentes para afron- } \\
\text { tar el reto educativo desde la innovación, en } \\
\text { donde las TIC ocupan un papel fundamental } \\
\text { (dimensión 1). }\end{array}$ & $\begin{array}{l}\text { - Proyectos y programas de innovación educa- } \\
\text { tiva que ofrece la administración para afrontar } \\
\text { el reto de la comunicación lingüística y las } \\
\text { TIC desde una visión de centro (dimensiones } \\
4 \text { y 5). } \\
\text { - La existencia de aplicaciones móviles (app), } \\
\text { estrategias (booktuber, booktrailer...) y de } \\
\text { plataformas virtuales con materiales para el } \\
\text { fomento y disfrute de la lectura contribuyen a } \\
\text { un nuevo modelo de aprendizaje autónomo } \\
\text { y autorregulado (m-learning, b-learning...) en } \\
\text { torno al estudiante (dimensión 5). }\end{array}$ \\
\hline
\end{tabular}

Figura 5. Matriz DAFO sobre uso de las TIC en el fomento de la lectura 
Tras este análisis, queremos indicar que no se han pretendido generalizar los resultados, sino plantear un foro de debate en el que han emergido las reflexiones de los verdaderos destinatarios de la formación inicial: los futuros docentes de lengua y literatura. La lectura de este trabajo puede abrir nuevos caminos e ideas que pueden replicarse en diversos contextos educativos.

\section{REFERENCIAS BIBLIOGRÁFICAS}

Amar, V. M. (2008). Tecnologías de la Información y la Comunicación, Sociedad y Educación. Sociedad, e-herramientas, profesorado y alumnado. Madrid: Tébar.

Argote, P. y Molina. M. A. (2010). Familia y escuela: influencia en la formación de lectores para el mañana. Aproximación a la lectura preadolescente y adolescente. Fundación la Fuente.

Baelo, R. y Cantón, I. (2009). Las tecnologías de la información y la comunicación en la educación superior. Revista Iberoamericana de Educación, 50(7), 1-12.

Barberá, E. (2003). La educación en la red. Actividades virtuales de enseñanza y aprendizaje. Barcelona: Paidós.

Bautista, S. y Méndez, M. (2015). Prácticas de lectura y escritura mediadas por las TIC en contextos educativos rurales. Revista Científica Guillermo de Ockham, 13(1), 97-107.

Boixareu, M. (2014). La mediación lectora: una responsabilidad de la familia, de la escuela, y también del Estado. RAÍDO, 8(17), 81-86.

Bozu, Z. e Imbernón, F. (2016). La formación docente en momentos de cambios ¿Qué nos dicen los profesores principiantes universitarios? Profesorado: Revista de curriculum y formación del profesorado, 20(3), 467-492.

Cabero, J. (2000). Las nuevas tecnologías en la Sociedad de la Información. En J. Cabero (Coord.), Nuevas tecnologías aplicadas a la educación (pp. 1-20). Madrid: Síntesis.

Cabero, J. (2005). Las TIC y las Universidad: retos, posibilidades y preocupación. Revista de la Educación Superior, 34(3), 77-100.

Caldera, R., Escalante, D. y Terán, M. (2010). Practica pedagógica de la lectura y formación docente. Revista Pedagogía, 31(88), 15-37. Recuperado de https://bit. ly/2Tdtmf3.

Casado, R. (2006). Alfabetización digital: qué es y cómo debemos entenderla. En R. Casado (Coord.), Claves de la alfabetización digital. Fundación Telefónica. Materiales presentados en las Jornadas sobre Alfabetización Digital (pp. 67-73). Barcelona: Ariel.

Cassany, D. (2012). En_línea. Leer y escribir en la red. Barcelona: Anagrama.

Cassany, D. y Llach, S. (2017). La digitalización del aula de ciencias: creencias y prácticas. Digital Education Review, 31, 93-115. 
Cerrillo, P. C., Larrañaga, E. y Yubero, S. (2002). Libros, lectores y mediadores. La formación de los hábitos lectores como proceso de aprendizaje. Cuenca: Ediciones de la Universidad de Castilla-La Mancha.

Cobo, J. C. (2011). El concepto de tecnologías de la información. Benchmarking sobre las definiciones de las TIC en la sociedad del conocimiento. Zer-Revista de Estudios de Comunicación, 14(27), 295-318.

Colás, P. (1998). La investigación-acción. En P. Colás y L. Buendía, Investigación educativa (pp. 250-260). Sevilla: Alfar.

Cuevas, A. y Marzal, M. Á. (2007). La competencia lectora como modelo de alfabetización en información. Anales de documentación, 10, 49-70.

Duso, L.M. y Cerutti, E. (2017). Docencia y cultura digital: la formación de ciberprofesor. Tendencias pedagógicas, 30, 207- 226.

Gallar, Y., Rodríguez, I. E. y Barrios, E. A. (2015). La mediación con las TIC en el proceso de enseñanza-aprendizaje de la educación superior. Revista Didasc@lia: Didáctica y Educación, 6(6), 155-164.

García, R., Rebollo, Á y Garcia, F. F. (2016). Relación Entre las preferencias de formación del profesorado y su competencia digital en las redes sociales. Bordón. Revista de pedagogía, 68(2), 137-153. https://doi.org/10.13042/Bordon.2016.68209.

Garrido-Lora, M., Busquet, J. y Munté-Ramos, R-À. (2016). De las TIC a las TRIC: estudio sobre el uso de las TIC y la brecha digital entre adultos y adolescentes en España. Anàlisi: Quaderns de comunicación i cultura, 54, 44-57. http://doi. org/10.7238/a.v0i54.2953.

Gil, J. y Martil-Carmona, R. (2018). El empoderamiento del alumnado a través de las TRIC. Creaciones narrativas a través de 'stop motion' en educación primaria. Index. comunicación. Revista científica de comunicación aplicada, 8(2), 189-210.

Gross, M. y Stiller, L. (2015). Contribución de la técnica del grupo focal al acercamiento a la percepción estudiantil sobre accesibilidad en el entorno universitario. Revista Actualidades Investigativas en Educación, 15(1), 1-16. http://doi. org/10.15517/aie.v15i1.17587.

Heredia, H. y Romero, M. F. (2019). Técnicas para la evaluación de la lectura y las TIC: tres cuestionarios para su diagnóstico. Campo Abierto. Revista de Educación, 38(1), 45-63. Recuperado de https://mascvuex.unex.es/revistas/index.php/campoabierto/article/view/3154.

Katz, R. (2009). El papel de las TIC en el desarrollo. Barcelona: Ariel.

Lluch, G. (2014). Jóvenes y adolescentes hablan de lectura en red. OCNOS: revista de estudios sobre lectura, 11, 7-20. https://doi.org/10.18239/ocnos_2014.11.01.

Marqués, P. (2000a). La cultura de la sociedad de la información. Aportaciones de las TIC. Recuperado de goo.gl/Byjhsk. 
Marquès, P. (2000b): Competencias básicas en la sociedad de la información. Alfabetización digital. Roles de los estudiantes hoy. Recuperado de goo.gl/O4apT2.

Martín, G. (2000). La lectura y los niños. El País. Recuperado de goo.gl/6dfMbN.

Milena, N., González, A. C., Torres, A.C. y Araya, J. (2017). Alfabetización digital a padres de familia en el uso de las redes sociales. Alteridad, 12(1), 8-19.

Milena, S. (2014). Rol mediador docente en la compresión de textos. Enunciación, 19(2), 252-267. https://doi.org/10.14483/udistrital.jour.enunc.2014.2.a06.

Mur, L. (2016). La nueva brecha digital. El futuro de las nuevas tecnologías en Primaria desde la formación del profesorado. Revista electrónica interuniversitaria de formación del profesorado, 19(2), 301-313. https://doi.org/10.6018/ reifop.19.2.189561.

Palamidessi, M. (2006). Las escuelas y las tecnologías, en el torbellino del nuevo siglo. En M. Palamidessi (Comp.), La escuela en la sociedad de redes. Una introducción a las tecnologías de la información y la comunicación en la educación (pp. 13-31). Buenos Aires: Fondo de Cultura Económica.

Palomo, R., Ruiz, J. y Sánchez, J. (2003). Marco de la experiencia. En Las Tic como agentes de innovación educativa (pp. 7-20). Junta de Andalucía.

Paredes, J. (2005). Animación a la lectura y TIC: creando situaciones y espacios. Revista de Educación, número extraordinario, 255-279.

Romero, M. F. y Jiménez, R. (2014). El practicum del MAES y la formación inicial en la enseñanza de lengua: entre la realidad y el deseo. Lenguaje y textos, 34, 49-58.

Romero, M. F. y Trigo, E. (2015). Herramientas para el éxito. Cuadernos de Pedagogía, 458, 16-21.

Sadín, M. ${ }^{a}$ Paz (2003). Investigación cualitativa en educación: fundamentos y tradiciones. Madrid: McGraw-Hill España.

Sáez, J. M. (2010). Análisis de la aplicación efectiva de la metodología en la práctica pedagógica en general y en el uso de las TICs en particular. XXI. Revista de educación, 12, 261-272.

Sainz, L. Ma (2005). La importancia del mediador: una experiencia en la formación de lectores. Revista de educación, 1, 357-362.

Santiago, M., Romero, M. Fco. y Goenechea, C. (2019). De los medios a las aulas. Actitudes de los futuros profesores de secundaria hacia la libertad de expresión y la participación en entornos digitales. Revista Espacios, 40(2), 1-13. Recuperado de https://bit.ly/2HjcBKk.

Santos, I. C. (2017). Desarrollo curricular y recursos educativos en las bibliotecas escolares. Investigaciones Sobre Lectura, 7, 36-54.

Semenov, A. (2005). Las tecnologías de la información y la comunicación en la enseñanza. Manual para docentes o Cómo crear nuevos entornos de aprendizaje 
abierto por medio de las TIC. Organización de las Naciones Unidas, para la Educación, la Ciencia y la Cultura. Montevideo, Uruguay: Trilce.

Stake, R. E. (1995). The art of case study research. Thousand Oaks, CA: Sage.

Tello, E. (2008). Las tecnologías de la información y comunicaciones (TIC) y la brecha digital: su impacto en la sociedad de México. Revista de Universidad y Sociedad del Conocimiento, 4(2), 1-8. Recuperado de https://bit.ly/2MemoCx.

Trigo, E. (2016). El papel de la formación inicial y permanente del profesorado para forjar lectores. RESED. Revista de Estudios Socioeducativos, 4, 66-84.

Trujillo, F. y Conecta 13 (2014). Artefactos digitales: Una escuela digital para la educación de hoy. Barcelona: Graó.

Unión Internacional de Telecomunicaciones (2003). Informe sobre el desarrollo mundial de las Telecomunicaciones, UIT.

Yubero, S. (1996). Animación a la lectura en diversos contextos. En P. C. Cerrillo y J. García (Coords.) Hábitos lectores y animación a la lectura (pp. 60-70). Cuenca: Ediciones de la Universidad de Castilla-La Mancha.

Zayas, F. (2010). 10 ideas clave. La competencia lectora según PISA. Reflexiones y orientaciones didácticas. Barcelona: Graó. 